\title{
Artículo
}

\section{La evaluación del alumnado universitario en el Espacio Europeo de Educación Superior}

\author{
Sonia San Martín Gutiérrez ${ }^{\mathrm{a}, *}$, Nadia Jiménez Torres $^{\mathrm{a}}$ y Estefanía Jerónimo Sánchez-Beato ${ }^{\mathrm{b}}$ \\ a Departamento de Economía y Administración de Empresas, Facultad de CC. Económicas, Universidad de Burgos, Burgos, España \\ ${ }^{\mathrm{b}}$ Departamento de Ciencias Sociales, Facultad de Ciencias Sociales, Universidad Europea Miguel de Cervantes, Valladolid, España
}

\section{INFORMACIÓN DEL ARTÍCULO}

\section{Historia del artículo:}

Recibido el 23 de enero de 2015

Aceptado el 27 de marzo de 2015

On-line el 20 de mayo de 2015

\section{Palabras clave:}

Enseñanza superior

Evaluación

Guías docentes

Alumnado

Profesorado

\begin{abstract}
R E S U M E N
Las guías docentes de las asignaturas constituyen un contrato entre la universidad y el alumnado en el que se recogen las competencias que debe adquirir el alumnado, el temario de la asignatura y los sistemas de evaluación, entre otros aspectos. El objetivo de este trabajo es analizar los sistemas de evaluación reflejados en las guías docentes publicadas en distintos grados de cuatro universidades españolas. Al efecto, se ha recogido información de 552 guías docentes de esas cuatro universidades, tres públicas y una privada, y se han analizado -mediante análisis de frecuencias, medias y tabulaciones cruzadas- las pruebas incluidas y las diferencias por universidades y ramas de conocimiento. En contra de lo que cabría esperar en las evaluaciones del alumnado realizadas en el marco del Espacio Europeo de Educación Superior, pocas guías relacionan la evaluación con la adquisición de competencias; el examen final sigue siendo clave, al punto de que no es mayoritaria la aplicación del sistema de evaluación continua, y sistemas como la autoevaluación y la coevaluación son minoritarios en los sistemas de evaluación; se emplean poco las nuevas tecnologías en el proceso de evaluación y la mera asistencia a clase constituye un sistema de evaluación en las guías.
\end{abstract}

(C) 2015 Instituto de Ciencias de la Educación de la Universidad de Oviedo. Publicado por Elsevier España, S.L.U. Este es un artículo Open Access bajo la licencia CC BY-NC-ND (http://creativecommons.org/licenses/by-nc-nd/4.0/).

\section{The evaluation of the university students in the European Higher Education Area}

\section{A B S T R A C T}

Subject teaching guides constitute a contract between the university and the students that includes, among other issues, the content of the course, assessment systems and competencies that students must have. The objective of this study is to analyze assessment systems reflected in the teaching guides published for different degrees by four Spanish universities. To do this, information has been collected from 552 teaching guides from these 4 universities, 3 of them public universities and one a private university, and an analysis performed using frequencies, means, contingency tables, the tests included, the differences by universities, and branches of knowledge. Contrary to what would be expected of the student evaluations made in the European Higher Education Area environment, only a few of the teaching guides associate evaluations with competencies. The final examination continues to be key; thus the application of continuous evaluation is not in general use, with self-assessment and co-evaluation being in the minority as evaluation systems. New technologies are not widely used in the student evaluation, and the mere attendance to a class is an evaluation system reflected in several teaching guides.

(c) 2015 Instituto de Ciencias de la Educación de la Universidad de Oviedo. Published by Elsevier España, S.L.U. This is an open access article under the CC BY-NC-ND license (http://creativecommons.org/licenses/by-nc-nd/4.0/).

\footnotetext{
* Autor para correspondencia: Calle Parralillos, s/n 09001 Burgos España, Tel.: +947258950; fax: +947258956. Correo electrónico: sanmargu@ubu.es (S. San Martín Gutiérrez).
} 


\section{Introducción}

Los sistemas de evaluación constituyen uno de los elementos básicos en la planificación y ejecución del proceso de enseñanzaaprendizaje (De Miguel, 2005), al tiempo que uno de los elementos curriculares que más dificultades plantea en el desarrollo docente (Trigueros, Rivera y de la Torre, 2012). Asimismo, se trata de una de las cuestiones que más afectada se ha visto por el proceso de convergencia hacia el Espacio Europeo de Educación Superior (en adelante, EEES) (López, 2006). Para conocer los sistemas elegidos por el profesorado universitario, resulta muy útil su estudio a través de las guías docentes. Dejando en este trabajo a un lado las valoraciones subjetivas del alumnado y profesorado, se considera que las guías docentes publicadas para cada asignatura suponen un contrato entre la universidad y el alumnado, en el que se puede incluir, con mayor o menor grado de detalle, distintos aspectos, tales como los objetivos, el índice del temario, las competencias que debe adquirir el alumnado, la forma de impartición de las clases, los resultados esperados del aprendizaje, y lo que interesa en el presente trabajo: las pruebas y sistemas de evaluación así como los pesos otorgados a cada sistema de evaluación.

La investigación docente ha abordado las características que debe reunir un buen profesorado (Casero, 2010; Celdrán y Escartín, 2008; Gargallo, Sánchez, Ros y Ferreras, 2010; Martínez, García y Quintanal, 2006; Voss y Gruber, 2006; Zabalza, 2003). No obstante, es un tema no resuelto pues la actividad docente es una tarea compleja, multidimensional, en la que el profesorado puede adoptar enfoques diferentes respecto a la enseñanza, lo que explica la falta de unanimidad a la hora de delimitar qué es una docencia de calidad (Ruiz, 2005). El EEES conlleva cambios en el proceso educativo que afectan al perfil del profesorado universitario, como el papel clave del alumnado en su propio proceso educativo (López-Sidro, 2011). El EEES implica formas de aprendizaje, recursos y tipos de evaluación distintos o modificados (Álvarez et al., 2009; RodríguezIzquierdo, 2014). Centrando la idea para el objeto de estudio, a saber, la evaluación docente en el ámbito universitario, sintéticamente, esta no puede tratarse ni de una tarea de control externa, ni de un mero trámite burocrático-administrativo, sino de un proceso de intercambio y comprensión de información sobre los acontecimientos que tienen lugar en un aula universitaria y que están relacionados con el tipo de actividades que se llevan a cabo, con el tipo de materiales utilizados, con la metodología empleada por el profesor, con las inquietudes e intereses del alumnado, con las relaciones y el clima de clase, etc. Un proceso de evaluación por y para los protagonistas, es decir, llevado a cabo por el profesorado, contando siempre con las perspectiva del alumnado, con la finalidad de crear estrategias docentes que mejoren tanto el aprendizaje de los/as estudiantes como la formación de los/as docentes (Alcaraz, Fernández y Sola, 2012, p. 28).

El nuevo modelo de enseñanza-aprendizaje impone que estén alineados con las competencias tanto los métodos como los sistemas de evaluación (Castejón y Santos, 2011; De Miguel, 2005; Fernández, 2010; Rodríguez-Izquierdo, 2014). Este alineamiento con las competencias supone cambios importantes, y a todos los niveles, en la configuración de los sistemas de evaluación, dado que, además de servir para acreditar un aprendizaje, deben ayudar al alumnado a aprender y al profesorado a mejorar su docencia (Antón, 2012). Son cuatro los cambios más significativos que ello conlleva (vid. De Miguel, 2005). En primer lugar, la evaluación auténtica: la evaluación, lejos de ser un acto casual, marginal o parcial, debe constituir un acto planificado, integral y pertinente a las competencias a alcanzar, a las demandas que plantea el desempeño profesional; en segundo lugar, la evaluación referida al criterio: en una evaluación alineada con las competencias resulta necesario ir más allá de la pura definición de las competencias, estableciendo para cada una de ellas, o para cada conglomerado de competencias, los niveles de logro o desempeño que se consideran adecuados; en tercer lugar, el apoderamiento de la evaluación por parte de los alumnos: el nuevo paradigma focalizado en el aprendizaje del alumnado implica necesariamente un papel activo y responsable por parte de este y diluye la distinción entre lo que se puede considerar actividad de aprendizaje y actividad de evaluación; por último, la evaluación continua y formativa: las actividades de evaluación sumativas y finales deben complementarse con actividades de evaluación formativas y continuas.

Así, diversos autores (Alcaraz et al., 2012; López, 2006; Platero, Benito y Rodríguez, 2012) parten de concebir la evaluación como un proceso fundamentado en la recogida de información útil, relevante y que permite emprender espacios de mejora en los procesos de enseñanza-aprendizaje. Partiendo de este planteamiento, abogan por acercarse más a la comprensión e implicación del alumnado en el proceso educativo que tiene lugar en las aulas, con vistas a la mejora del aprendizaje, y por alejarse por completo de la intención medidora o sancionadora que a menudo se asocia a los procesos de evaluación, a modo de mera certificación del éxito o fracaso en los procesos de aprendizaje.

Si se analizan estudios previos, el sistema deseado por el alumnado es la evaluación continua y la presentación de trabajos. Gargallo et al. (2010) encuentran que los métodos de evaluación que los alumnos proponen como idóneos son, por este orden: valoración de trabajos (86\%), valoración del esfuerzo del alumno (68\%) y exámenes parciales-valoración de la realización de las prácticas (ambas respuestas con la misma frecuencia, 54\%). También son elegidos con frecuencias importantes la valoración de la asistencia a clase (48\%), la valoración del interés del alumno (46\%) y la valoración de las actividades diarias de clase (40\%). Ningún alumno considera como buen sistema de evaluación el basado solo en un examen final. Un estudio previo que se ha realizado con alumnado preuniversitario justo cuando llegan a la universidad, revela que entre las formas de evaluación preferidas por el alumnado español destacan los exámenes parciales a lo largo del curso $(72,4 \%)$, la entrega de trabajos sin exámenes (70\%) y la realización de trabajos en grupo (64,3\%). Entre las menos valoradas aparecen con un $8,6 \%$ la realización de un único examen al final del curso y los exámenes orales con un $7 \%$. Cuando se les pide que escojan un máximo de tres aspectos a valorar en el sistema de evaluación ideal del profesorado universitario eligen en las tres primeras posiciones, y muy distanciadas del resto, la posibilidad de recuperar solo las partes no superadas $(71,4 \%)$ y valorar el esfuerzo realizado durante el curso (67,9\%).

Se pueden clasificar las vías de aprendizaje y de evaluación en tres grandes grupos (Fraile, López, Castejón y Romero, 2013). En primer término, se tendría la vía continua. Esta implica la utilización de un sistema de evaluación formativa y continua, que aporta feedback al alumnado para su aprendizaje. No suele haber examen final y si lo hay supone solo una parte de la calificación global. La calificación final proviene de las actividades de evaluación realizadas durante el aprendizaje. El alumnado debe asistir de forma continuada, entregar documentos en plazo y forma, corregir los errores señalados por el profesorado y superar los niveles básicos establecidos para las actividades de evaluación). Se promueve la participación del alumnado en la evaluación, mediante las técnicas de autoevaluación, evaluación entre iguales o evaluación compartida. Este sistema de evaluación es el que se encuentra en consonancia con la evaluación en la docencia universitaria en el marco del EEES.

En segundo lugar, estaría la vía mixta. Esta se basa en el aprendizaje durante el cuatrimestre y una prueba final, con mayor peso en la calificación que la vía anterior. Los requisitos son menos exigentes que en la vía continua, por ejemplo: asistencia más o menos regular (en torno al 50\% de las sesiones), o realización obligatoria del trabajo grupal, pero voluntaria del resto de actividades de aprendizaje. La calificación final proviene de combinar la prueba final y las actividades de aprendizaje. Es flexible y admite 
diferentes situaciones. Es una vía que, con frecuencia, elige el alumnado en situaciones especiales (laborales o enfermedad, entre otras), pero que también existe en la evaluación ordinaria.

Finalmente, se encuentra la vía examen final. Este modelo de evaluación-calificación tradicional requiere superar un examen final con pruebas (entre otras, teórica, práctica, proyecto), para valorar el grado de dominio de las competencias establecidas en la asignatura. La calificación se obtiene de sumar cada una de las pruebas finales. Aprobar la convocatoria supone hacerlo por separado para cada una de las pruebas. Esta vía es necesario ofrecerla para cumplir con los reglamentos de ordenación académica de muchas universidades, que establecen que el alumnado tiene derecho a una convocatoria de evaluación. Normalmente esta vía debería ser la utilizada por el alumnado que no asiste regularmente a clase, o bien por el alumnado que no ha realizado las actividades de aprendizaje obligatorias de la vía mixta.

Algunos autores, como Nicol y Macfarlane-Dick (2006), llegan a proponer un conjunto de principios para que el alumnado sea la parte esencial en su evaluación, se implique y participe de forma muy activa en su aprendizaje y evaluación. De igual modo, Carless, Joughin y Liu (2006) señalan que el alumnado debe ser gestor de su aprendizaje y el profesorado universitario debe ser un orientador y facilitador del mismo, lo que adquiere gran importancia en sistemas de evaluación como la coevaluación y la autoevaluación (Ibarra, Rodríguez y Gómez, 2012; Trujillo, 2011). También un estudio de Fraile et al. (2013), realizado sobre 52 asignaturas de 19 universidades españolas en las que el docente aplica sistemas de evaluación formativa, muestra que la utilización de dichos sistemas influye positivamente en la mejora del rendimiento académico del alumnado, especialmente en lo referente a un elevado porcentaje de "aptos» y un bajo porcentaje de abandonos («no presentados»). Si se compara el rendimiento académico en función de la vía de aprendizaje y evaluación elegida por el alumnado, los datos parecen mostrar un rendimiento académico notablemente más alto en las vías continua y mixta que en la vía examen final, como reflejo de un mejor proceso de aprendizaje (Fraile et al., 2013).

En esta misma línea, un estudio descriptivo llevado a cabo por Castejón y Santos (2011), realizado con el primer curso del Grado de Ciencias de la Actividad Física y del Deporte (en adelante, CAFD) en el departamento universitario de la Universidad Autónoma de Madrid mediante los datos recogidos en las guías docentes y las entrevistas al profesorado y al alumnado, revela que la metodología participativa y la evaluación formativa son aspectos claves en la puesta en práctica de los grados en el EEES. Específicamente, en la formación inicial de los titulados en CAFD supone un cambio de orientación en el aprendizaje del alumnado desde la perspectiva del profesorado y de ese mismo alumnado.

Otro estudio realizado con una muestra de 65 estudiantes que han cursado las asignaturas que se analizan comparativamente con dos métodos distintos (tradicional versus coevaluación), muestra que la coevaluación añade valor al aprendizaje del alumnado, si bien dicho valor no necesariamente se ve reflejado en los resultados académicos. Los resultados que este trabajo arrojaron fueron que, en las asignaturas estudiadas -aunque los datos sobre los rendimientos académicos eran mejores aplicando el sistema de evaluación tradicional-, la coevaluación reducía el absentismo en el aula y añadía valor a la formación mediante el desarrollo de habilidades competenciales (Platero et al., 2012). Además, no se debe olvidar la utilización de recursos electrónicos y nuevas tecnologías utilizados ampliamente en la docencia actual, en la interacción profesoradoalumnado y en la evaluación correspondiente, mediante recursos como Moodle, MUD, o foros. (Ellis, Goodyear, Prosser y O'Hara, 2006; Fisher y Baird, 2005; Thomas, 2002).

Para analizar los principales cambios experimentados por los sistemas de evaluación a raíz de la implantación del EEES, una pieza clave de análisis la constituyen las guías docentes. En este sentido, existen trabajos que señalan las pautas para elaborarlas (GarcíaSanz, 2008; Zabalza y Zabalza, 2012). Entre ellos, Zabalza y Zabalza (2012) proponen un modelo de guía docente que constituye un auténtico instrumento de orientación y diálogo con el alumnado y que debe incluir los datos generales, su sentido curricular, sus propósitos formativos, los contenidos de la propuesta, las líneas metodológicas en las que se mueve y, por lo que en este trabajo interesa, el sistema de evaluación utilizado. Además, estos autores proponen que la construcción de un buen programa docente constituye una competencia básica de los buenos profesores, aquellos a los que no les basta con conocer bien el campo disciplinar, sino que saben cómo combinar ese conocimiento con las particulares condiciones en las que deben llevar a cabo su docencia. Por ello, sostienen que las guías deben ser mecanismos que se asemejen a contenedores abiertos y flexibles en los que los docentes van incorporando progresivamente todos los nuevos avances y conocimientos que consideren relevantes.

Debido a los escasos trabajos sobre evaluación del alumnado universitario en el EEES, el análisis de las guías docentes supone una importante aportación a la literatura sobre evaluación. Es cierto que ya se encuentran entre la doctrina algunos estudios enfocados hacia los nuevos sistemas de evaluación docente a través del examen de las guías didácticas (entre otros, Castejón y Santos, 2011; Lazcano-Peña, 2013). No obstante, estos se centran en determinadas ramas de conocimiento -CAFD, Comunicación-, en algunos casos, en estudios desarrollados sobre las guías de una única universidad, e incluso otros centrados en el análisis de una cuestión específica dentro de los sistemas de evaluación (p. ej. la importancia de los sistemas de evaluación participativa y formativa).

En esta línea, este estudio pretende realizar un análisis, de forma comparativa, de numerosas guías docentes, de distintas ramas de conocimiento, de todos los grados ofertados por cuatro universidades españolas. El objetivo de este trabajo es analizar los sistemas de evaluación reflejados en las guías docentes publicadas en distintos grados de esas cuatro universidades, comparando asimismo universidades y ramas de conocimiento. La hipótesis de partida es que las nuevas metodologías docentes implantadas desde el EEES han supuesto una amplia variedad, y cantidad, de sistemas de evaluación, si bien se podrán apreciar ciertas similitudes entre los sistemas elegidos por cada rama de conocimiento, al partir de competencias semejantes, así como entre los sistemas establecidos desde cada universidad, por existir una serie de protocolos propios a cada una de ellas en esta materia.

\section{Método}

\section{Muestra}

Para llevar a cabo el estudio empírico, los miembros del grupo de innovación docente Innovación Multidisciplinar Aplicada a los Grupos de Enseñanza (I.M.@.G.E.), con profesorado perteneciente a cuatro universidades diferentes (tres públicas y una privada), buscaron y analizaron las guías docentes publicadas en cada universidad en todos los grados que se imparten (grados ya adaptados al EEES). En concreto, se analizaron las guías docentes de 263 asignaturas de la Universidad de Valladolid (pública), 130 asignaturas de la Universidad de Burgos (pública), 107 asignaturas de la Universidad Pablo de Olavide (Sevilla) (pública) y 52 asignaturas de la Universidad Europea Miguel de Cervantes (Valladolid) (privada). La tabla 1 refleja algunas características básicas de las universidades cuyas guías docentes se han analizado.

Se utilizó una muestra de 552 guías docentes (todas ellas publicadas), seleccionadas por un sistema de muestreo probabilístico, eligiéndose la $5 .^{\mathrm{a}}$ guía correspondiente a una asignatura obligatoria de cada curso dentro de cada grado y la 5. a asignatura optativa 
Tabla 1

Datos de las universidades de la muestra y guías docentes analizadas en este trabajo

\begin{tabular}{|c|c|c|c|c|}
\hline Universidad & $\begin{array}{l}\text { Número de grados } \\
\text { (excluidos dobles } \\
\text { grados) }\end{array}$ & $\begin{array}{l}\text { Número de grados } \\
\text { analizados en este } \\
\text { trabajo (\%) }\end{array}$ & $\begin{array}{l}\text { Número de } \\
\text { asignaturas }\end{array}$ & $\begin{array}{l}\text { Número (y \%) de } \\
\text { guías analizadas }\end{array}$ \\
\hline Universidad de Burgos & 27 & $27(100)$ & 1.274 & $130(10,2)$ \\
\hline Universidad de Valladolid & 52 & $52(100)$ & 2.978 & $263(8,8)$ \\
\hline Universidad Pablo de Olavide & 18 & $18(100)$ & 603 & $107(17,7)$ \\
\hline Universidad Europea Miguel de Cervantes & 12 & $12(100)$ & 429 & $52(12,1)$ \\
\hline
\end{tabular}

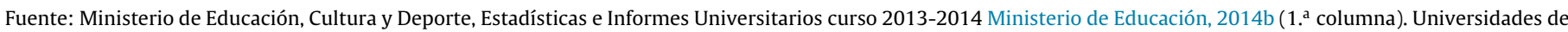
la muestra (3..$^{\mathrm{a}}$ columna). Nuestro trabajo (2. ${ }^{\mathrm{a}}$ y $4 .^{\mathrm{a}}$ columnas).

de último curso de grado (del listado de asignaturas ordenado del plan de estudios ofrecido en la página web de cada universidad analizada), por lo que en la muestra objeto de estudio al menos se incluye una guía de todos los cursos de todos los grados de cada una de las universidades elegidas (ver tabla 1). En concreto, el 20\% de las guías son de primer curso, el $21 \%$ son de segundo curso, el $26 \%$ de tercer curso, el $32 \%$ de cuarto curso y un $1 \%$ de quinto curso. El $60 \%$ correspondía a asignaturas de tipo obligatorio, el $21 \%$ a optativas y el $19 \%$ a asignaturas básicas. Además, cabe señalar que 133 guías (24\%) son de grados correspondientes a la rama de ciencias sociales y jurídicas, 125 guías (23\%) son de ciencias o ciencias de la salud, 104 guías (19\%) son de humanidades y 190 guías (34\%) son de ingenierías. De este modo, se puede observar que representan bien los datos poblacionales por ramas dado que, según datos del Ministerio de Educación, Cultura y Deporte (2014), la distribución por ramas de los grados verificados es la siguiente: $35 \%$ de los grados pertenecen a la rama de ciencias sociales y jurídicas, el $16 \%$ a humanidades, el $22 \%$ a ciencias y ciencias de la salud y el $27 \%$ a ingeniería y arquitectura. La recogida y análisis de datos se realizó durante los meses de enero, febrero y marzo de 2014.

\section{Medición y variables}

Para realizar el estudio empírico, se han realizado diversas reuniones entre los miembros del grupo de innovación docente así como con otros profesores consultados, tras las cuales se decidió analizar las siguientes variables de cada guía docente seleccionada y en las que están contempladas las características de todas las guías analizadas. En primer lugar, se observaron datos tales como universidad, grado, departamento, asignatura y curso.

Asimismo, se analizó si se distinguen o no sistemas de evaluación, si los ponderan, si se marcan mínimos para pasar cada prueba de evaluación, si se relacionan sistemas de evaluación con competencias, si las pruebas de la convocatoria ordinaria y extraordinaria son las mismas o no. Estas variables son dicotómicas y se codificaron como 1 en caso afirmativo y 2 en caso negativo para cada característica (ej. si distingue sistemas de evaluación se introdujo un 1 y si no distingue, se introdujo un 2 en la variable correspondiente).

Por último, se examinó si se evalúa mediante examen final, examen parcial, pruebas de desarrollo, pruebas tipo test, examen oral final, un trabajo global final, un conjunto de prácticas parciales, varias pruebas orales, la participación en clase, la asistencia en clase, portafolios, autoevaluación, recursos electrónicos u otros sistemas. Todas estas variables se trataron como dicotómicas igual que las anteriores (ej. si se utiliza el examen final como sistema de evaluación se introdujo un 1 y si no se contempla el examen final se introdujo un 2). También se contemplaron los pesos otorgados a cada sistema de evaluación utilizado (en porcentajes) cuando se han ponderado los sistemas en las guías docentes analizadas.

\section{Procedimiento}

La información contenida en las guías fue registrada por los miembros del grupo de innovación docente siguiendo el esquema acordado de variables introducido por todos en el programa SPSS según una hoja de registro que incluyó todos los apartados contenidos en las guías. Cada miembro introdujo todos los datos de la misma variable para las distintas universidades con el fin de conseguir una homogeneidad en la introducción de los datos. Además, un miembro del grupo que no introdujo datos revisó todas las guías introducidas y se pusieron en común en el grupo las pocas dudas que existieron al respecto. Todas las guías docentes utilizadas son públicas y difundidas en las correspondientes páginas web de cada universidad. El análisis de todos los datos de las guías docentes fue realizado con el programa estadístico SPSS versión 18.

\section{Análisis de datos}

En primer lugar, se revisó la base de datos para comprobar que no había datos anómalos o ausentes que pudieran distorsionar los resultados. En segundo lugar, con los datos introducidos, se realizaron análisis univariantes (frecuencias) y bivariantes (tablas de contingencia para cruzar datos y evaluar la significación de las relaciones bivariadas mediante los estadísticos Chi-cuadrado y V de Cramer) (Hair, Anderson, Tatham y Black, 2001; Novales, 2003).

\section{Resultados}

Como aspectos generales de los sistemas de evaluación recogidos en las guías analizadas, el 94,5\% distingue sistemas de evaluación, el $90,2 \%$ ofrece la ponderación o pesos de cada sistema de evaluación, el $60 \%$ señala mínimos para pasar cada prueba de evaluación y solo un 5,9\% relaciona los sistemas de evaluación con la adquisición de competencias. En el 34\% de las guías docentes se señalan sistemas de evaluación diferentes para la convocatoria ordinaria y la extraordinaria, un $21 \%$ indican los mismos sistemas en ambas convocatorias y un $44,9 \%$ no hace alusión a la convocatoria extraordinaria.

La tabla 2 resume los sistemas concretos de evaluación incluidos en las guías docentes analizadas y entre los que destaca el examen final (77,9\%). Por el contrario, algunos de los sistemas de evaluación más recomendados, como la coevaluación, son contemplados explícitamente por muy pocas guías. El $15 \%$ incluye sistemas de evaluación mencionados de forma muy aislada y normalmente corresponden a campos específicos ligados a un grado concreto o solo vienen contemplados en una guía docente en nuestra muestra. Se advierte que la suma total de guías y de porcentajes correspondientes a los sistemas de evaluación utilizados puede ser mayor que 552 guías y que el $100 \%$ en cada guía docente (p. ej. una guía puede señalar evaluación mediante examen final con un peso del $30 \%$ y después indicar evaluación tipo test con un peso del $20 \%$ sin señalar el otro $10 \%$ restante a qué prueba de evaluación corresponde, por lo que en la base de datos aparece examen final 30\% y examen test $20 \%)$.

En cuanto a los pesos otorgados a cada prueba de evaluación, y dado que la variabilidad entre guías en algunos casos es grande (ej. el peso del examen final varía desde nada hasta un $100 \%$ ), se ha calculado el peso medio otorgado a cada sistema de evaluación, 
Tabla 2

Sistemas de evaluación incluidos en las guías docentes

\begin{tabular}{|c|c|c|}
\hline Número de guías & Porcentaje de guías & Sistema de evaluación \\
\hline 430 & 77,9 & Examen final \\
\hline 392 & 71 & $\begin{array}{l}\text { Se valora el conjunto de varias } \\
\text { prácticas parciales }\end{array}$ \\
\hline 237 & 42,9 & Participación activa en clase \\
\hline 160 & 28,9 & Exámenes parciales \\
\hline 160 & 28,9 & $\begin{array}{l}\text { Entrega de un trabajo final o global } \\
\text { de asignatura }\end{array}$ \\
\hline 138 & 25 & Examen de desarrollo \\
\hline 127 & 23 & Varias pruebas orales \\
\hline 99 & 17,9 & Examen tipo test \\
\hline 83 & 15 & Asistencia a clase \\
\hline 33 & 5,9 & Portafolio \\
\hline 28 & 5 & Examen oral \\
\hline 22 & 3,9 & Uso de recursos electrónicos \\
\hline 11 & 1,9 & Autoevaluación \\
\hline 83 & $\begin{array}{l}15 \text { (otros sistemas } \\
\text { o pruebas de } \\
\text { evaluación) }\end{array}$ & $\begin{array}{l}\text { Coevaluación, debates, herbario, } \\
\text { prácticas de laboratorio, valoración } \\
\text { cualitativa, trabajo de campo, } \\
\text { seminarios, pósters, asistencia a } \\
\text { conferencias, comentarios de texto } \\
\text { o imágenes, participación en un } \\
\text { juicio }\end{array}$ \\
\hline
\end{tabular}

Fuente: Elaboración propia.

de modo que el examen final tiene un peso medio de $52 \%$, el examen parcial un $23,9 \%$, el de desarrollo un $21,9 \%$, el test un $13,9 \%$, la prueba final oral un 5,9\%, el trabajo escrito global un $15,9 \%$, las prácticas parciales un $27 \%$, las pruebas orales un $11 \%$, la participación un $13,9 \%$, la asistencia a clase un $4 \%$, el portafolio un $4 \%$, la autoevaluación un $0,9 \%$, los recursos electrónicos un $2,9 \%$ y otras pruebas un $23,9 \%$.

Además, se han realizado comparaciones entre las guías de las distintas universidades incluidas en la muestra y se han observado diferencias significativas (tabla 3). En concreto, se puede señalar que en las guías docentes de la universidad privada de la muestra (Universidad Europea Miguel de Cervantes), siempre se ponderan los sistemas de evaluación y es la que más marca mínimos para pasar una prueba de evaluación (82,7\%), es donde más se utilizan exámenes parciales, pruebas de desarrollo y pruebas tipo test. En la universidad privada de la muestra no destaca la asistencia y participación en clase como sistema de evaluación específico (solo en un $7,9 \%$ de las guías incluye la participación en clase y el 5,9\% la asistencia a clase como sistema de evaluación), quizás porque se da por supuesta en la docencia en el marco del EEES. Además, en estas guías se señalan sistemas de evaluación que no se han encontrado en las guías de las universidades públicas, tales como las escalas de actitudes o las fichas de observación sistemáticas (34,6\%). Las guías de la Universidad Pablo de Olavide son las que más señalan la asistencia a clase $(24,3 \%)$ y las guías de la Universidad de Burgos son las que más incluyen la participación en clase como prueba evaluable $(60,1 \%)$. En las universidades públicas el examen final sigue siendo un sistema de evaluación muy utilizado (por encima del $80 \%$ de las guías en las tres universidades públicas analizadas). De ellas, la Universidad de Valladolid es la que menos utiliza pruebas parciales y orales como sistemas de evaluación.

Finalmente, se ha tratado de analizar si existían diferencias significativas por ramas de conocimiento (tabla 4). El análisis por ramas muestra que las disciplinas de ciencias sociales son aquellas en las que el examen final tiene mayor importancia (91,7\%). En el caso de las ciencias, son los que menos utilizan examen final, utilizan más tests, pruebas orales en medicina y recurren a sistemas específicos de la rama (como, por ejemplo, las prácticas de laboratorio o el herbario) (19,2\%) y normalmente es diferente la evaluación ordinaria de la extraordinaria (41,6\%). Tanto en ciencias sociales como en ciencias se utiliza el examen de desarrollo y el tipo test en mayor medida que en las otras ramas de conocimiento. En la rama de humanidades, prevalecen más que en otros campos sistemas de evaluación como las pruebas orales $(32,7 \%)$ y la participación en clase $(64,4 \%)$. Además, no suelen señalar el sistema de evaluación utilizado en la convocatoria extraordinaria. Finalmente, en el campo de las ingenierías es donde más se utilizan recursos electrónicos, aunque son poco utilizados en las evaluaciones del alumnado. Además, son los que menos valoran la participación en clase $(30 \%)$ y donde más prevalece el examen parcial como sistema de evaluación (41,1\%). Se parecen a los grados de la rama de ciencias en la inclusión de sistemas de evaluación específicos de las materias de esos campos.

\section{Discusión}

Tal y como se ha planteado al inicio de este trabajo, el objetivo era analizar las guías docentes publicadas de cuatro universidades diferentes para conocer los sistemas de evaluación que se están aplicando en los grados adaptados al EEES y conocer así el grado de aplicación del sistema de evaluación continua, las nuevas formas de evaluación del alumnado y los pesos otorgados a cada sistema de evaluación, entre otros aspectos. De hecho, como se señalaba como hipótesis de partida, se comprueba que las nuevas metodologías docentes implantadas desde el EEES han supuesto una amplia variedad, y cantidad, de sistemas de evaluación, aunque se pueden apreciar ciertas similitudes entre los sistemas elegidos por cada rama de conocimiento, así como entre los sistemas establecidos desde cada universidad.

El trabajo presentado aborda los sistemas de evaluación del alumnado universitario según las guías docentes publicadas en la docencia impartida en los grados adaptados al EEES. Aunque hay estudios como el de Gargallo et al. (2010) que resalta la conveniencia de valorar el esfuerzo del alumnado y ese es el espíritu de la docencia en el EEES, se ha podido observar que el examen final sigue teniendo un peso importante en la evaluación. De igual forma, se puede señalar el contraste entre la importancia de sistemas de evaluación tan útiles como la autoevaluación (2\%) o la coevaluación, como destacan Ibarra et al. (2012) o Trujillo (2011), y la escasa importancia que se les otorga en las guías docentes de la muestra analizada. Así, con la autoevaluación, el alumnado se implica, se motiva y reflexiona sobre su propio aprendizaje, comprende mejor el rol del profesorado, interactúa con el profesorado, y en la coevaluación, interactúa con sus compañeros (Carless et al., 2006). En efecto, en el contexto de la formación por competencias, no solamente las personas responsables de la formación intervienen en el proceso de evaluación, sino que la autoevaluación del alumnado es crucial, puesto que la capacidad de emitir un juicio sobre su propio progreso es parte integrante de todas las competencias. Además, la capacidad de reflexionar del alumno sobre lo que ha conseguido y aquello que no ha logrado es la piedra angular de un gran número de enunciados de competencias. La evaluación compartida, colaborativa o coevaluación por su parte hace referencia a los procesos de diálogo que mantiene el profesorado con el alumnado sobre la evaluación de los aprendizajes y los procesos de aprendizaje-enseñanza que tienen lugar (Fernández, 2010). Todos estos procesos evaluativos tienen su fundamentación pedagógica en el desarrollo de procesos de la denominada uevaluación democrática», que parte de la convicción de que la vida en democracia es algo que se aprende y que requiere una serie de hábitos, competencias y responsabilidades (López, 2006), y un ámbito en el que estos deben aplicarse es en la evaluación, favoreciendo dinámicas de participación, responsabilidad y compromiso con el proceso de aprendizaje, del alumnado (Trigueros et al., 2012).

Dado que los componentes de las competencias a evaluar son de muy diferente naturaleza (conocimientos, habilidades y 
Tabla 3

Comparación de los sistemas de evaluación entre las guías docentes de las distintas universidades $(\mathrm{N}=552)$

\begin{tabular}{|c|c|c|c|c|c|c|c|}
\hline \multirow[t]{2}{*}{ Sistemas de evaluación } & \multicolumn{4}{|c|}{ Universidad de (en \%) } & \multirow[t]{2}{*}{$\chi^{2}$} & \multirow[t]{2}{*}{ Sig. } & \multirow[t]{2}{*}{$\mathrm{V}$} \\
\hline & $\begin{array}{l}\text { Valladolid } \\
\text { (pública) }\end{array}$ & $\begin{array}{l}\text { Burgos } \\
\text { (pública) }\end{array}$ & $\begin{array}{l}\text { Pablo de Olavide } \\
\text { (Sevilla) (pública) }\end{array}$ & $\begin{array}{l}\text { Europea Miguel } \\
\text { de Cervantes } \\
\text { (privada) }\end{array}$ & & & \\
\hline $\begin{array}{l}\text { Ponderan sistemas de } \\
\text { evaluación }\end{array}$ & 89,2 & 98,5 & 97,2 & 100 & 19,93 & $<0,001$ & 0,19 \\
\hline $\begin{array}{c}\text { Señalan mínimos para pasar } \\
\text { una prueba de evaluación }\end{array}$ & 43,8 & 86,3 & 69,2 & 82,7 & 77,46 & $<0,001$ & 0,38 \\
\hline $\begin{array}{l}\text { Incluye examen final como } \\
\text { sistema de evaluación }\end{array}$ & 85,4 & 80,6 & 86 & 21,1 & 112,53 & $<0,001$ & 0,32 \\
\hline $\begin{array}{l}\text { Incluye examen parcial como } \\
\text { sistema de evaluación }\end{array}$ & 21,5 & 40,3 & 24,3 & 53,8 & 29,37 & $<0,001$ & 0,24 \\
\hline $\begin{array}{l}\text { Incluye pruebas de desarrollo } \\
\text { como sistema de evaluación }\end{array}$ & 16,1 & 1,9 & 37,4 & 94,2 & 177,75 & $<0,001$ & 0,59 \\
\hline $\begin{array}{l}\text { Incluye prueba tipo test como } \\
\text { sistema de evaluación }\end{array}$ & 12,3 & 12,2 & 30,8 & 36,5 & 31,85 & $<0,001$ & 0,25 \\
\hline $\begin{array}{l}\text { Incluye prácticas parciales } \\
\text { como sistema de evaluación }\end{array}$ & 59,2 & 80,6 & 80,4 & 84,6 & 31,96 & $<0,001$ & 0,24 \\
\hline $\begin{array}{l}\text { Incluye diversas pruebas orales } \\
\text { como sistema de evaluación }\end{array}$ & 15,4 & 35,4 & 25,2 & 32,7 & 21,17 & $<0,001$ & 0,20 \\
\hline $\begin{array}{l}\text { Incluye participación en clase } \\
\text { como sistema de evaluación }\end{array}$ & 36,9 & 60,1 & 55,1 & 7,9 & 49,45 & $<0,001$ & 0,31 \\
\hline $\begin{array}{l}\text { Incluye asistencia a clase como } \\
\text { sistema de evaluación }\end{array}$ & 10,8 & 17,9 & 24,3 & 5,9 & 14,75 & $<0,001$ & 0,17 \\
\hline $\begin{array}{l}\text { Incluye otros sistemas de } \\
\text { evaluación minorita-rios (ej. } \\
\text { escalas de actitudes) }\end{array}$ & 14,6 & 9,5 & 10,3 & 34,6 & 21,17 & $<0,001$ & 0,20 \\
\hline $\begin{array}{l}\text { Evaluación extraordinaria } \\
\text { distinta de la ordinaria }\end{array}$ & 33,1 & 23,6 & 34,6 & 67,3 & 99,27 & $<0,001$ & 0,31 \\
\hline Número total de guías & 130 & 263 & 107 & 52 & & & \\
\hline
\end{tabular}

Fuente: Elaboración propia.

Tabla 4

Comparación de los sistemas de evaluación entre las distintas ramas de conocimiento $(\mathrm{N}=552)$

\begin{tabular}{|c|c|c|c|c|c|c|c|}
\hline Sistemas de evaluación & $\begin{array}{l}\text { Ciencias sociales y } \\
\text { jurídicas (\%) }\end{array}$ & Ciencias (\%) & Humani-dades (\%) & Ingenie-ría (\%) & $\chi^{2}$ & Sig. & $\mathrm{V}$ \\
\hline $\begin{array}{l}\text { Incluye examen final como } \\
\text { sistema de evaluación }\end{array}$ & 91,7 & 66,4 & 87,5 & 72,1 & 34,22 & $<0,001$ & 0,18 \\
\hline $\begin{array}{l}\text { Incluye examen parcial como } \\
\text { sistema de evaluación }\end{array}$ & 25,6 & 28,8 & 16,3 & 41,1 & 19,19 & $<0,001$ & 0,19 \\
\hline $\begin{array}{l}\text { Incluye examen de desarrollo } \\
\text { como sistema de evaluación }\end{array}$ & 36,1 & 38,4 & 4,8 & 20 & 41,28 & $<0,001$ & 0,29 \\
\hline $\begin{array}{l}\text { Incluye un examen tipo test } \\
\text { como sistema de evaluación }\end{array}$ & 24,8 & 24,8 & 2,9 & 17,4 & 21,93 & $<0,001$ & 0,21 \\
\hline $\begin{array}{l}\text { Incluye un examen oral como } \\
\text { sistema de evaluación }\end{array}$ & 10,5 & 4 & 7,7 & 2,1 & 13,18 & $<0,001$ & 0,11 \\
\hline $\begin{array}{l}\text { Incluye diversas pruebas orales } \\
\text { como sistema de evaluación }\end{array}$ & 17,3 & 27,2 & 32,7 & 19,5 & 10,29 & $<0,001$ & 0,14 \\
\hline $\begin{array}{l}\text { Incluye participación en clase } \\
\text { como sistema de evaluación }\end{array}$ & 40,6 & 40,8 & 64,4 & 30 & 30,13 & $<0,001$ & 0,24 \\
\hline $\begin{array}{l}\text { Incluye evaluación mediante } \\
\text { recursos electrónicos como } \\
\text { sistema de evaluación }\end{array}$ & 1,5 & 2,4 & 1,9 & 7,9 & 10,38 & $<0,001$ & 0,14 \\
\hline $\begin{array}{l}\text { Incluye otros sistemas de } \\
\text { evaluación minoritarios (ej. } \\
\text { laboratorio, herbario) }\end{array}$ & 10,5 & 19,2 & 4,8 & 19,5 & 13,86 & $<0,001$ & 0,17 \\
\hline $\begin{array}{l}\text { Evaluación extraordinaria } \\
\text { distinta de la ordinaria }\end{array}$ & 22,6 & 41,6 & 25,9 & 38,9 & 26,09 & $<0,001$ & 0,16 \\
\hline Número total de guías & 133 & 125 & 104 & 190 & & & \\
\hline
\end{tabular}

Fuente: Elaboración propia.

destrezas, actitudes y valores), el alineamiento de la evaluación con las competencias obliga al uso combinado y hasta integrado de diferentes estrategias y procedimientos. De este modo, la evaluación de competencias se basaría en el acceso a fuentes múltiples y variadas de información con el fin de determinar si el alumnado ha alcanzado el nivel esperado de desarrollo de competencias. Así pues, en un mismo curso deberán realizarse actividades evaluativas de tipo sumativo y final (garantistas y posiblemente comparativas) y actividades evaluativas formativas y continuas. Finalmente, la evaluación debe integrarse, incluso camuflarse, dentro del proceso de enseñanza y aprendizaje y también debe ser viable que el alumnado se autoadministre la evaluación (De Miguel, 2005; Fernández, 2010).

Las tecnologías de la información y la comunicación permiten al alumnado llevar a cabo un aprendizaje mucho más colaborativo con sus compañeros y profesores gracias a la comunicación interactiva, tal y como indicaban también los trabajos de Álvarez et al. (2009) y de De Pablo y Villaciervos (2005). Sin embargo y aunque el alumnado valora la utilización de las nuevas tecnologías por parte del profesorado (Gargallo et al., 2010), en las pruebas de 
evaluación solo un 4\% las utiliza. Finalmente, llama la atención que se contemple explícitamente la asistencia a clase como un sistema de evaluación, probablemente algo que se supone obligatorio en la docencia actual, y que no habría que puntuar si no hay la correspondiente participación activa del alumnado en clase. En todo caso, lo anterior confirma, como se adelantaba en la hipótesis inicial de este trabajo, la variedad y cantidad de sistemas de evaluación que ha supuesto la implantación del Plan Bolonia.

Asimismo, se observan diferentes pruebas de evaluación por cada una de las cuatro universidades analizadas, y según la rama del conocimiento. En efecto, cada universidad posee su normativa en cuanto a aspectos como la elaboración de las guías y los pesos máximos de los sistemas de evaluación utilizados para cada asignatura, siendo posible adelantar cierta comparación entre ellas. En las universidades públicas analizadas, el examen final sigue teniendo un papel relevante en la evaluación de las asignaturas, pese a que cabría esperar una mayor aplicación de la evaluación continua. En unas universidades se observa que se marcan mínimos para cada prueba en mayor medida que en otras. En la muestra de la universidad privada no se distingue la asistencia y participación en clase como sistema de evaluación específico, quizás porque se dé por supuesta en la docencia en el marco del EEES, y se señalan sistemas de evaluación que no se han encontrado en las guías de las universidades públicas, tales como las escalas de actitudes o las fichas de observación sistemáticas.

El análisis por ramas muestra que, en las disciplinas de ciencias sociales utilizan más tests, examen de desarrollo y el examen final como sistemas de evaluación. En el caso de las ciencias, se han encontrado más guías que marcan mínimos, son las que menos utilizan examen final, utilizan más tests y recurren a sistemas específicos de la rama (como, por ejemplo, las prácticas de laboratorio o el herbario) y normalmente es diferente la evaluación ordinaria de la extraordinaria. En la rama de humanidades, prevalecen más que en otros campos sistemas de evaluación como las pruebas orales, prácticas parciales y participación en clase; además, no suelen señalar el sistema de evaluación utilizado en la convocatoria extraordinaria. En el campo de las ingenierías, se encuentran las guías que más utilizan el examen parcial y recursos electrónicos, en comparación con las otras ramas de conocimiento. Se puede resumir que las guías docentes del campo de humanidades muestran más diferencias en evaluación que otras ramas de conocimiento, en tanto que hay puntos en común de las ramas de ciencias sociales y ciencias. Así, en el campo de humanidades se aprecia una preferencia especial por pruebas de carácter oral (pruebas orales, participación en clase), y por el contrario, un escaso recurso a sistemas de evaluación como el test o los recursos electrónicos, cuestiones ambas que, en cierta medida, también se presentan en las ramas de ciencias sociales y jurídicas.

Como limitaciones del presente estudio, se puede reconocer el hecho de que este trabajo se haya centrado en el análisis de las guías, las cuales no necesariamente reflejan lo que ocurre en el aula. Además, la muestra compuesta por las cuatro universidades en las que se ha basado el estudio no ha sido elegida aleatoriamente, razón por lo cual, aunque tienen características muy dispares entre sí (de tamaño, naturaleza pública o privada, titulaciones ofertadas, entre otras) se ha de ser cautelosos a la hora de generalizar los resultados obtenidos a otras universidades y contextos.

Como futuras líneas de continuación y mejora de este trabajo, cabe señalar que el objetivo último es poder comparar los sistemas de evaluación publicados en las guías docentes, las opiniones y preferencias del alumnado y el profesorado universitario para obtener una comparación integral de los agentes implicados en la evaluación del alumnado en el marco del EEES. Asimismo, se considera que sería interesante diseñar una guía docente básica y analizar si podría ser utilizada, de manera transversal, para asignaturas pertenecientes a distintas ramas de conocimiento.

\section{Financiación}

Financiación recibida de la Universidad de Burgos para la realización del proyecto de innovación y mejora docente en que se enmarca este trabajo (proyecto 2.2., resolución de julio de 2013 para la convocatoria del curso 2012-2013 para dos cursos académicos).

\section{Conflicto de intereses}

Los autores declaran no tener ningún conflicto de intereses.

\section{Agradecimiento}

Nos gustaría poner de manifiesto nuestro agradecimiento a la Universidad de Burgos, donde está registrado el grupo I.M.@.G.E., por la financiación recibida para la realización del proyecto de innovación y mejora docente en que se enmarca este trabajo (proyecto 2.2., resolución de julio de 2013 para la convocatoria del curso 2012-2013 para dos cursos académicos) y a todos los profesores que amablemente han decidido colaborar en este trabajo y han mostrado su interés.

\section{Referencias bibliográficas}

Alcaraz, N., Fernández, M. y Sola, M. (2012). La voz del alumnado en los procesos de evaluación docente universitaria. Revista Iberoamericana de Evaluación Educativa, 5(2), 26-39.

Álvarez, V., Asensio, I., Clares, J., del Frago, R., García Lupión, B., García, N., et al. (2009). Perfiles y competencias docentes requeridos en el contexto actual de la educación universitaria. Revista Española de Orientación y Psicopedagogía, 20(3), 270-283.

Antón, M. A. (2012). Docencia universitaria: concepciones y evaluación de los aprendizajes. Estudio de casos [tesis doctoral]. Burgos: Universidad de Burgos.

Carless, D., Joughin, G., Liu, N. F., \& Associates. (2006). How assessment supports learning: Learning-oriented assessment in action. Hong Kong: Hong Kong University Press.

Casero, A. (2010). ¿Cómo es el buen profesor universitario según el alumnado? Revista Española de Pedagogía, 246, 223-242.

Castejón, F. J. y Santos, M. L. (2011). Percepciones y dificultades en el empleo de metodologías participativas y evaluación formativa en el Grado de Ciencias de la Actividad Física. Revista Electrónica Interuniversitaria de Formación del Profesorado, 14(4)

Celdrán, M. y Escartín, J. (2008). ¿Qué piensan los alumnos universitarios sobre las competencias interpersonales de su profesorado? Un estudio cualitativo. Univest, 08, 1-13.

De Miguel, M. (Dir.) (2005). Modalidades de enseñanza centradas en el desarrollo de competencias. Orientaciones para promover el cambio metodológico en el Espacio Europeo de Educación Superior. Oviedo: Ministerio de Educación y Ciencia. Universidad de Oviedo.

De Pablo, J. y Villaciervos, P. (2005). El Espacio Europeo de Educación Superior y las tecnologías de la información y la comunicación. Percepciones y demandas del profesorado. Revista de Educación, 337, 99-124.

Ellis, R. A., Goodyear, P., Prosser, M. y O'Hara, A. (2006). How and what university students learn through online and face-to-face discussion: Conceptions, intention and approaches. Journal of Computer Assisted Learning, 22, 244-256.

Fernández, A. (2010). La evaluación orientada al aprendizaje en un modelo de formación por competencias en la educación universitaria. Revista de Docencia Universitaria, 8(1), 11-34.

Fisher, M. y Baird, D. E. (2005). Online learning design that fosters student support, self-regulation, and retention. Campus-Wide Information Systems, 22(2), 88-107.

Fraile, A., López, V. M., Castejón, F. J. y Romero, R. (2013). La evaluación formativa en docencia universitaria y el rendimiento académico del alumnado. Aula Abierta, 41(2), 23-34

García-Sanz, M. P. (2008). Guías docentes de asignaturas de grado en el EEES. Orientaciones para su elaboración. Murcia: Editum.

Gargallo, B., Sánchez, F., Ros, C. y Ferreras, A. (2010). Estilos docentes de los profesores universitarios. La percepción de los alumnos de los buenos profesores. Revista Iberoamericana de Educación, 51(4), 1-16.

Hair, J. F., Anderson, R. E., Tatham, R. L. y Black, W. C. (2001). Análisis multivariante. Madrid: Prentice Hall.

Ibarra, M. S., Rodríguez, G. y Gómez, M. A. (2012). La evaluación entre iguales: beneficios y estrategias para su práctica en la universidad. Revista de Educación, 359, 206-231.

Lazcano-Peña, D. (2013). Metodologías de investigación: aproximación a su enseñanza en el grado de periodismo en España. Periodística, 15, 11-28.

López, V.M.(2006). El papel de la evaluación formativa en el proceso de convergencia hacia el EEES. Análisis del estado de la cuestión y presentación de un sistema de intervención. Revista Interuniversitaria de Formación del Profesorado, 20(3), 93-119. 
López-Sidro, A. (2011). Reflexiones sobre los desafíos de Bolonia y la misión del profesor universitario. Entre la innovación y la tradición. Revista Jurídica de Investigación e Innovación Educativa, 4, 23-44.

Martínez, M., García, B. y Quintanal, J. (2006). El perfil del profesor universitario de calidad desde la perspectiva del alumno. Educación XX1, 9, 183-198.

Ministerio de Educación, Cultura y Deporte (2014). Datos básicos del sistema universitario español, curso 2013-2014 [consultado 27 Feb 2015]. Disponible en: http://www.mecd.gob.es/dms/mecd/educacion-mecd/areas-educacion/ universidades/estadisticas-informes/datos-cifras/DATOS_CIFRAS_13_14.pdf

Ministerio de Educación, Cultura y Deporte (2014). Estadísticas e Informes Universitarios [consultado 27 Feb 2015]. Disponible en: http://www.mecd.gob.es/ educacion-mecd/areas-educacion/universidades/estadisticas-informes.html

Nicol, D. y Macfarlane-Dick, D. (2006). Formative assessment and self-regulated learning: A model and seven principles of good feedback practice. Studies in Higher Education, 31(2), 199-218.

Novales, A. (2003). Manual de econometría. Madrid: McGraw Hill.

Platero, M., Benito, S. y Rodríguez, A. (2012). Co-evaluación y asignación de roles, una experiencia de innovación docente universitaria. Docencia e Investigación, 22, 7-29.
Rodríguez-Izquierdo, R. M. (2014). Modelo formativo en el Espacio Europeo de Educación Superior: valoraciones de los estudiantes. Aula Abierta, 42 $106-113$.

Ruiz, J. (2005). La evaluación de la docencia en los planes de mejora de la universidad. Educación XX1, 8, 87-102.

Thomas, M. J. W. (2002). Learning within incoherent structures: The space of online discussion forums. Journal of Computer Assisted Learning, 18, 351-366.

Trigueros, C., Rivera, E. y de la Torre, E. (2012). La evaluación en el aula universitaria: del examen tradicional a la autoevaluación. Revista Internacional de Medicina y Ciencias de la Actividad Física y del Deporte, 47, 473-491.

Trujillo, E. (2011). La autoevaluación como acción tutorial en la formación integra del estudiante universitario. Pampedia, 7, 44-56.

Voss, R. y Gruber, T. (2006). The desired teaching qualities of lecturers in higher education: A means end analysis. Quality Assurance in Education, 14(3), 217-242.

Zabalza, M. A. (2003). Competencias docentes del profesorado universitario. Madrid: Narcea.

Zabalza, M. A. y Zabalza, M. A. (2012). Planificación de la docencia en la universidad. Elaboración de las guías docentes de las materias. Madrid: Narcea. 